\section{LA-UR- $95-4180$}

Title:

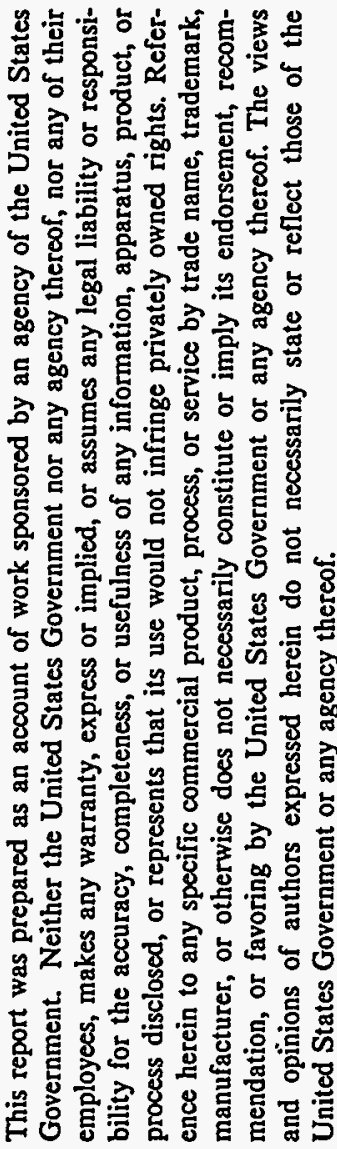

Submitted to:

Author(s):

\section{Directly Labeled Fluorescent DNA Probes for Chromosome Mapping}

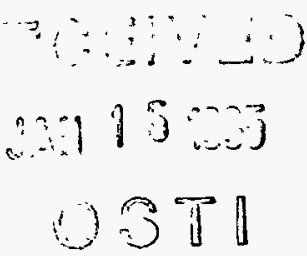

Babetta L. Marrone, LS-5

Larry L. Deaven, CHGS

David J. Chen, LS-6

Min S. Park, LS-6

Mark A. Macinnes, LS-6

Gary C. Salzman, LS-5

Thomas M. Yoshida, CST-9

\section{DOE Office of Scientific and Technical Information (OSTI)}

\section{Los Alamos}

NATIONAL LABORATORY

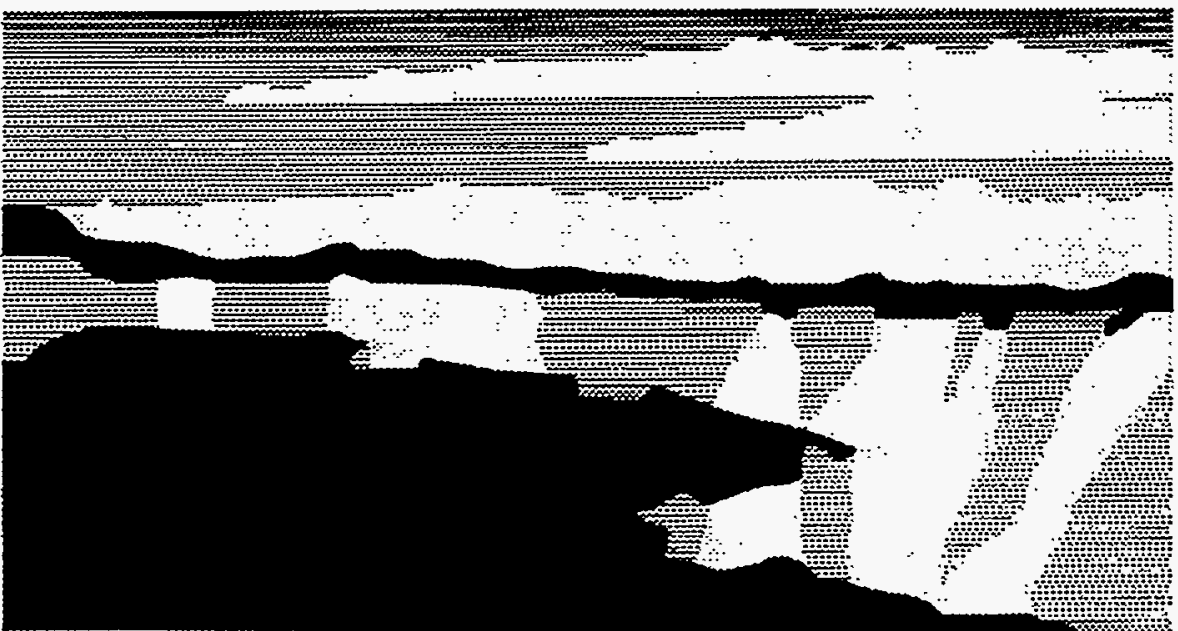

Los Alamos Nalional Laboratory, an aftimative action/equal opportunity employer, is operated by the University of Calfomia for the U.S. Department of Energy under contract W-7405-ENG-36. By acceptance of this article, the publisher recognizes that the U.S. Government retains a monexclusive, royaltyfree Ilcense to publlsh or reproduce the published form of this contribution, or to allow others to do so, for U.S. Govermment purposes. The Los Alamos Nalional Laboratory requests that the publisher Identlfy this article as work performed under the auspices of the U.S. Department of Energy. 


\title{
Directly Labeled Fluorescent DNA Probes for Chromosome Mapping
}

\author{
Babetta L. Marrone*, Larry L. Deaven, David J. Chen, Min S. Park, Mark A. MacInnes, \\ Gary C. Salzman, and Thomas M. Yoshida
}

\begin{abstract}
This is the final report of a three-year Laboratory-Directed Research and Development (LDRD) project at the Los Alamos National Laboratory (LANL). The project outlines a new strategy for employing nucleic acid probes that are directly labeled with fluorochromes in fluorescence in situ hybridization techniques. These probes will permit the detection, quantitation, and highprecision spatial analysis of multiple DNA sequences along a single chromosome using video-enhanced fluorescence microscopy and digital image processing and analysis. Potential advantages of direct labeled DNA probes for fluorescence in situ hybridization far surpass currently available, indirect DNA probe labeling techniques in ease of use, versatility, and increased signal-tonoise ratio.
\end{abstract}

\section{Background and Research Objectives}

The fluorescent labeling of biomolecules has advantages over widely used radioisotopic labeling techniques in terms of increased user safety, faster sample analysis, and the potential for monitoring multiple probes on the same cell or chromosome in one assay by multi-spectral analysis. The most widely used technique for fluorescent labeling of DNA probes is an indirect technique and relies on the high binding affinity of avidin for biotin. Nucleotides are modified with a linker arm terminating in biotin and are incorporated into probe DNA using chain elongation or nick translation. The biotinylated probe is reacted with the specimen after a denaturing step to make the target DNA or mRNA accessible. Fluorescently labeled avidin is then applied, whereupon it binds to biotin on the probe. Detection consists of $35 \mathrm{~mm}$ microphotography to document the presence and approximate location of probe binding. Alternatively, microscope-based digital imaging is used to precisely localize and quantitate the fluorescence. Variations in this indirect labeling technique have been made with the goal of

*Principal investigator, e-mail: blm@lanl.gov 
increasing the number of probes that are analyzed simultaneously in the same specimen. The variations entail a modification of the probe DNA with, e.g. aminoacetyl-fluorene, sulfonate, mercury-hapten, or digoxigenin attached through a linker arm. In each case the modified group is reacted with an antibody; the primary antibody is then detected with a fluorescently labeled antiimmunoglobin antibody. Biotin-avidin and a combination of antigen-antibody complexes have been used to localize as many as four different probes on regions of chromosomes $(1,2)$.

Direct labeled fluorescent nucleotides (i.e. covalent attachment of a fluorochrome to a nucleotide), have significant advantages in the following areas over using indirect, biotinavidin or antibody techniques to fluorescently label DNA probes.

a) Low Background: Background fluorescence due to adventitious sticking of proteinconjugated fluorochromes is eliminated; a higher signal-to-noise ratio is expected.

b) High Sample Throughput: Fewer handling steps will accelerate in situ hybridization procedures and improve sample integrity.

c) Improved Quantitation: Fluorochrome number on the DNA probe is controllable; with a known number of fluorochromes per probe, fluorescence intensity is directly proportional to target sequence copy number.

d) High Resolution: Potentially enhanced spatial resolution by use of more compact probes.

e) Good Sensitivity: Since the cooled CCD camera allows photon-limited detection, detector sensitivity is not a limiting factor. Sensitivity will ultimately be limited by the amount of background and autofluorescence present. As a result, sensitivity may be equal to that in indirect methods.

We investigated a strategy for direct fluorescent labeling of nucleotide probes that has important advantages over current methods for application to high resolution, multispectral in situ hybridization studies in chromosomes or cells. Advanced fluorescence methodologies (both chemistry and instrumentation) were exploited to engineer optimal fluorescent DNA probes for quantitative fluorescence analysis following digital microscopy.

The overall objective of the research was to develop fluorescence in situ hybridization (FISH) probe technology to optimize the regional localization and to place in order multiple DNA sequences along a single chromosome using high precision image analysis. The immediate biological target of the proposed studies is the mapping of yeast artificial chromosomes (YACs) containing cloned fragments of selected human chromosomes onto their respective locations on these chromosomes. This work contributes toward integration of the physical and cytogenetic maps. The goals are multidisciplinary: 1) the development and application of biochemistry and molecular biological techniques to prepare and characterize labeled DNA probes; 2) the development and refinement of cooled CCD microscopy 
capabilities for quantitative, high-resolution, high-precision, multi-spectral image processing, and analysis of FISH probes; and 3) the development of software methods to facilitate and semi-automate chromosome mapping.

\section{Importance to LANL's Science and Technology Base and National R\&D Needs}

The research has broad relevance to several bioscience areas of major thrust at LANL and to national health needs. It :

a) Facilitates DNA mapping and sequencing efforts of the Human Genome Program by improving capabilities for nonradioisotopic detection of DNA probes. For chromosome mapping, direct-labeled DNA probes will greatly improve the speed at which samples can be analyzed, since time- and reagent-consuming post-hybridization/immunological amplification steps will be eliminated. Multi-spectral imaging will result in more information obtained from the same sample. Expected results will also accelerate progress on the Rapid DNA Sequencing project.

b) Enhances cell and radiation biology initiatives by developing multicolor, direct mode labeling techniques that will greatly facilitate the simultaneous assessment of multiple gene regulation events in single cells.

c) Contributes to structural biology efforts, since direct-labeled fluorescent DNA probes will improve spatial resolution of probe(s) located strategically on chromatin.

d) Provides technological improvements that enrich quantitative fluorescence microscopy capabilities and image cytometry for the biosciences.

\section{Scientific Approach and Results to Date}

Our approach incorporates labeled nucleotides into nucleic acid probes, which are then hybridized in situ to their target sequences on chromosomes. The location of the FISH probes on the chromosome are mapped using digital imaging microscopy to produce a high-resolution, high-precision map of selected human chromosomes by FISH.

Mapping of the fluorescent DNA probe to its location on the chromosome in a metaphase spread is done using a procedure modified slightly from the one described originally by Lichter et al. (3) for mapping of cosmid clones to chromosome 11 by FISH and digital imaging microscopy. YAC probes are indirectly labeled with fluorescein, and the total DNA of the chromosome is counterstained with propidium iodide. One image is acquired for each chromosome of interest (such as chromosome 21) containing the fluorescent probe signal in a 
metaphase spread. From the digitized image, a line is drawn with the cursor through the long axis of the chromosome of interest so that it passes through the fluorescent signal from the probe located on it. The relative photon intensity in each pixel along this line is displayed and the map position of the probe is expressed as the fractional length (FL) of the total chromosome relative to a fixed reference point which by convention is designated FLpter. Multiple (20-30) chromosomes/images are analyzed from each hybridization. Using this technique on metaphase chromosomes it is possible to map probes on chromosomes with a 1-2 Mb resolution.

Fractional length mapping of FISH probes was applied to several projects in the Life Sciences (LS) Division in FY95, including the mapping of cosmids in the region of chromosome 16 containing the gene for the inherited disease Familial Mediterranean Fever; mapping the DNA double-strand repair gene XRCC4 on chromosome 5q13-14; mapping the DNA repair gene FEN-1 on chromosome 11q13; and the mapping of gap regions on chromosome 16. Multiple-color mapping was also successfully applied to higher resolution mapping efforts in each of these projects. The single-color fractional length analysis and multiple-color mapping are in routine use in our laboratory now and will continue to be applied to future chromosome and gene mapping efforts in LS Division.

Fluorescent dUTP has been incorporated into a trinucleotide repeat DNA sequence using the PRimed IN Situ labeling technique (PRINS, 4). The tandem expansion of certain trinucleotide DNA sequence repeats has been implicated as a common cause of human genetic disease. The PRINS technique is a fast $(2 \mathrm{~h})$, simple, one-step procedure for sequencespecific detection of nucleic acids in situ.. Mapping of the hybridization domains generated from the PRINS labeling is continuing on chromosome 1. The results suggest a correspondence between high density clusters of the trinucleotide repeat CAG and cytogenetic bands known to contain fragile sites where breakage may occur. The PRINS method is also being adapted for application to routine FISH analyses of monochromosomal cell hybrids and purity checks of flow-sorted chromosomes.

In FY95 we intensified efforts to develop new software to facilitate chromosome mapping. The quantitative procedure for fractional length measurements of FISH probes on metaphase chromosomes has been further automated and refined. In addition, software methods were developed for: resolving two closely spaced or overlapping FISH signals on metaphase chromosomes using mathematical centroid-finding techniques; and for facilitating assignment of FISH or PRINS signals to cytogenetic band locations. These programs will continue to be exercised and optimized as they are applied to future chromosome mapping projects. 
During FY95 we also expanded our application of quantitative fluorescence analyses to include the mapping of cell immunofluorescence from digitized images collected by confocal microscopy or using the cooled CCD microscope system. The cellular distribution of immunofluorescence staining of a DNA repair protein (XPG) and its analogs was quantified and compared in normal cells and in cells that had DNA breakage due to UV irradiation. Software programs were developed for immunofluorescence analysis in subcellular regions and for counting of aggregates of fluorescence signals in nuclei. These methods have been adopted by other researchers in LS Division who are applying them to their cell immunofluorescence analysis projects.

\section{References}

1. Nederiof, P. M. et al.. Cytometry 11: 126-131, 1990.

2. Trask, B. and D. Pinkel. Methods in Cell Biology, Flow Cytometry, pp. 383-400, Eds: Z. Darzynkiewicz and H. A. Crissman, Academic Press, Inc., San Diego, CA, 1990. 3. Lichter, P. et al. Science 247:64-69, 1990.

4. Koch, J., J. Hindkjaer, J. Mogensen, S. Kolvraa, and L. Bolund. Gen. Anal. Tech. 8:171-178, 1991. 